\title{
A novel isoform of the human mitochondrial complex I subunit NDUFV3
}

Marris G. Dibley ${ }^{1}$, Michael T. Ryan ${ }^{1}$ and David A. Stroud ${ }^{1,2}$

'Department of Biochemistry and Molecular Biology, Monash Biomedicine Discovery Institute, Monash University, 3800, Melbourne, Australia.

2Correspondence to: d.stroud@monash.edu, Tel: +61 39905 9629, Address: 23 Innovation Walk, Monash University, 3800 Melbourne, Australia.

\begin{abstract}
Human mitochondrial complex $\mathrm{I}$ is the first enzyme of the mitochondrial respiratory chain. Complex $\mathrm{I}$ is composed of 45 subunits, seven encoded by mitochondrial DNA while the remainder are encoded by nuclear DNA. All nuclear-encoded subunits are thought to be expressed as a single isoform. Here we reveal subunit NDUFV3 to be present in both the canonical $10 \mathrm{kDa}$ and a novel $50 \mathrm{kDa}$ isoform, generated through alternative splicing. Both isoforms assemble into complex I and their levels vary in different tissues. While the $50 \mathrm{kDa}$ isoform appears to be dominant in HEK293T cells, we find either isoform alone is sufficient for assembly of mature complex I. NDUFV3 represents the first known complex I subunit present in two functional isoforms.
\end{abstract}

\section{Keywords}

Mitochondria; NADH dehydrogenase; Complex I; OXPHOS; metabolism 


\section{Introduction}

The mitochondrion is a dual membrane bound organelle, concentrically comprised of an outer membrane, an aqueous inter-membrane space, an inner-membrane and a protein-rich matrix. Embedded in the inner-membrane, the $980 \mathrm{kDa}$ mammalian complex I (NADH:ubiquinone oxidoreductase) is the largest of four multimeric enzymes which comprise the respiratory chain, generating a proton gradient and driving production of ATP ${ }^{1,2}$. In humans, complex I biogenesis involves the assembly of 45 subunits encoded by two genomes $^{3,4}$, and a suite of at least 15 assembly factors ${ }^{5-8}$. Subunits initially coalesce into five transient modules, which in-turn combine in a stepwise fashion to form two large protein $a^{2} s^{8}$. The hydrophilic matrix arm protrudes into the center of the mitochondrion and incorporates both the NADH binding N-module and the ubiquinone binding Q-module. The highly hydrophobic membrane arm contains the proton translocating $\mathrm{P}$-module and intersects with the matrix arm at almost $90^{\circ}$, giving the enzyme its characteristic ' $L$ '-shape ${ }^{3,4,9}$. The complexity of this convergence, coupled with its fundamental role in ATP synthesis, makes aberrant complex I assembly a significant cause of disease. Mutations in genes encoding complex I subunits and assembly factors are the most common cause of all mitochondrial dysfunction ${ }^{7,10}$. Pathogenic mutations have been shown to manifest as various diseases including Leigh Syndrome, Leber's Hereditary Optic Neuropathy (LHON) as well as neurodegeneration and premature aging ${ }^{7,10-12}$.

Forming the enzymatic core of complex I are fourteen highly conserved proteins common to bacterial complex I. The remaining 31 human subunits (29 individual proteins and two copies of acyl carrier NDUFAB1) are not found in the bacterial structure and are therefore termed "accessory" or "supernumerary" subunits ${ }^{3,4,13}$. Using gene-editing technologies, we recently generated a panel of human embryonic kidney (HEK293T) cell lines, each lacking a single accessory subunit ${ }^{14}$. While most subunits were required for normal assembly and function of the enzyme, loss of a small number of subunits led to only minor defects in the assembly of an otherwise functional complex. Among these was NADH dehydrogenase [ubiquinone] flavoprotein 3 (NDUFV3) - or $10 \mathrm{kDa}$ subunit in the bovine nomenclature. In addition to unique peptides representing the canonical $10 \mathrm{kDa}$ isoform, we detected peptides specific to a novel $\sim 50 \mathrm{kDa}$ isoform of NDUFV3. Both proteins are predicted to be encoded by the NDUFV3 gene and produced through alternative splicing of a single transcript. 
Here, we characterize the $\sim 50 \mathrm{kDa}$ isoform of NDUFV3 (NDUFV3-L). We demonstrate that both NDUFV3-L and the canonical isoform (NDUFV3-S) are expressed in human and mouse cells. While NDUFV3-L is more abundant than NDUFV3-S in HEK293T cells, the stoichiometry of each isoform varies in mouse tissues. Both isoforms of NDUFV3 are imported into the mitochondrion, assemble into the mature complex I and are alone sufficient to restore full assembly the complex.

\section{Materials and methods}

Cell culture

HEK293T cell lines ${ }^{15}$ were cultured in Dulbecco's Modified Eagle Medium (DMEM) supplemented with $10 \%(\mathrm{v} / \mathrm{v}$ ) fetal calf serum (FCS), penicillin/streptomycin and $50 \mu \mathrm{g} / \mathrm{mL}$ uridine. For stable isotope labelling with amino acids in cell culture (SILAC) cell were cultured as previously described ${ }^{14-16}$. Briefly, high-glucose SILAC DMEM lacking lysine and arginine (Thermo-Fisher Scientific) was supplemented with $600 \mathrm{mg} / \mathrm{L}$ L-proline, $10 \mathrm{kDa}$ dialysed FCS (Sigma), penicillin/streptomycin and uridine as above. Medias additionally contained either normal "light" lysine and arginine (146 mg/l L-Lysine- $\mathrm{HCl}$ and $42 \mathrm{mg} / \mathrm{L}$-arginine-HCl) or their "heavy" counterparts (180 mg/l ${ }^{13} \mathrm{C}_{6}{ }^{15} \mathrm{~N}_{2}$-L-lysine-2 $\mathrm{HCl}$ and $44 \mathrm{mg} /{ }^{13} \mathrm{C}_{6}{ }^{15} \mathrm{~N}_{4}$-L-arginie- $\mathrm{HCl}$; Cambridge Isotope Laboratories). All cells were incubated at $37^{\circ} \mathrm{C}$ at an atmosphere of $5 \%$ $\mathrm{CO}_{2}$.

\section{Generation of cell lines}

A HEK293T knockout of both NDUFV3 isoforms (NDUFV3 ${ }^{\mathrm{KO}}$ ) was previously described ${ }^{14}$. The knockout of NDUFV3-L was generated using CRISPR/Cas9 as essentially as described $^{14}$. Briefly, an oligoduplex was produced specific for the guide RNA designed using the online tool $\mathrm{CHOPCHOP}{ }^{17}$. Primers $(100 \mu \mathrm{m})$ were phosphorylated with T4 Polynucleotide Kinase (New England Biolabs; $37^{\circ} \mathrm{C}$ for 30 minutes) and using a PCR cycler the oligoduplex was formed by gradual lowering of temperature $\left(95^{\circ} \mathrm{C}\right.$ for 5 minutes, ramp down to $25^{\circ} \mathrm{C}$ at $5^{\circ} \mathrm{C}$ /minute). Phosphorylated oligoduplexes were ligated into Bbsl (New England Biolabs) digested pSPCas9-GFP ${ }^{18}$ (a gift from F Zhang; Addgene \#48138). The resulting construct was transfected into HEK293T cells using Lipofectamine ${ }^{\circledR}$ LTX (ThermoFisher Scientific), and cells single cell-sorted according to GFP expression into 96 well plates. Cells were 
expanded and genomic DNA was isolated through lysis of $100 \mu \mathrm{g}$ cells in $1 \mathrm{~mL} \mathrm{SDS} / \mathrm{pK}$ lysis buffer (67 mM Tris-Cl pH 8.8, $50 \mu \mathrm{g} / \mathrm{mL} \mathrm{pK,} 6.7 \mathrm{mM} \mathrm{MgCl} 2,2 \mathrm{mM} \mathrm{SDS}$ ), incubation at $37^{\circ} \mathrm{C}$ for 1 hour followed by $80^{\circ} \mathrm{C}$ for 10 minutes. The CRISPR/Cas9 targeted region was amplified by PCR and ligated into pGEM-4Z (Promega). Individual alleles were screened for mutations by Sanger sequencing as described ${ }^{19}$.

\section{Isolation of mitochondria}

Mitochondria were isolated as per Acin Perez et al. ${ }^{20}$. Briefly, cultured cells were harvested and collected by centrifugation ( $800 \times \mathrm{g}, 5$ minutes, $4^{\circ} \mathrm{C}$ ) before re-suspension in 10 cell pellet volumes of Buffer $\mathrm{A}$ (83mM sucrose, $10 \mathrm{mM}$ HEPES-KOH pH 7.2) and homogenization in a drill fitted pestle for 30 strokes. An equal volume of Buffer $B(250 \mathrm{mM}$ sucrose, $30 \mathrm{mM}$ HEPES$\mathrm{KOH} \mathrm{pH7.2)} \mathrm{was} \mathrm{added} \mathrm{to} \mathrm{the} \mathrm{homogenate,} \mathrm{and} \mathrm{the} \mathrm{material} \mathrm{centrifuged} \mathrm{for} 5$ minutes at $1000 \times g\left(4^{\circ} \mathrm{C}\right)$. The supernatant was removed and subjected to centrifugation at $12,000 \times g$ for 2 minutes $\left(4^{\circ} \mathrm{C}\right)$. Crude mitochondria were washed and re-suspended in Buffer $\mathrm{C}(320 \mathrm{mM}$ sucrose, $1 \mathrm{mM}$ EDTA, $10 \mathrm{mM}$ Tris- $\mathrm{Cl} \mathrm{pH} 7.4$ ). The protein concentration was quantified with Pierce BCA protein Assay Kit (ThermoFisher Scientific). For isolation of mitochondria from mouse tissue, wild-type C57BI/6 mice were sacrificed and heart, liver and brain tissues harvested. Tissues were washed and re-suspended in Buffer A and incubated on ice for 15 minutes to facilitate swelling. Crude mitochondria were isolated and mitochondrial protein quantified as above.

\section{SDS-PAGE, BN-PAGE and immunoblotting}

SDS-PAGE was performed using Tris-Tricine gradient gels ${ }^{21}$ and the Novex® NuPAGE SDSPAGE gel system (ThermoFisher Scientific). Blue native (BN)-PAGE and two-dimensional (2D)-PAGE was performed as described previously ${ }^{22}$. Gels were transferred to polyvinylidene difluoride (PVDF) membranes using a Novex Semi-Dry Blotter (ThermoFisher) according to manufacturer's instructions. Horseradish peroxidase coupled mouse and rabbit secondary antibodies (Sigma) and Clarity ${ }^{\mathrm{TM}}$ enhanced ECL chemiluminescent substrate (BioRad) were used for detection on a ChemiDoc XRS+ Imaging System (Bio-Rad, Hercules, CA-USA). Primary antibodies raised in rabbits against NDUFS5 and NDUFA $9^{23}$ were generated inhouse. Antibodies against NDUFV3 (ProteinTech) and OXPHOS subunits ("Total-OXPHOS"; Abcam) were commercially obtained. 


\section{Mitochondrial protein import}

NDUFV3-S and NDUFV3-L were amplified using primers designed to introduce an SP6 promoter sequence (5'-ATTTAGGTGACACTATAGAA-3') immediately upstream of the reading frame. The resulting PCR products were purified with the Wizard ${ }^{\mathrm{TM}} \mathrm{SV}$ Gel Purification and PCR Clean-up System and used as a template for synthesis of mRNA using the Ambion $\circledast$ mMessage mMachine Kit (Thermo Scientific). In vitro translation was performed in the presence $\left[{ }^{35} S\right]$ methionine/cysteine (Pierce) using the Rabbit Reticulocyte Lysate System (Promega). Protein import was performed essentially as described ${ }^{23}$. Briefly, mitochondria were collected by centrifugation $\left(12,000 \times g, 5\right.$ minutes, $\left.4^{\circ} \mathrm{C}\right)$ and $50 \mu \mathrm{g}$ protein re-suspended in $100 \mu \mathrm{L}$ ice-cold import buffer ( $250 \mathrm{mM}$ sucrose, $5 \mathrm{mM}$ magnesium acetate, $80 \mathrm{mM}$ potassium acetate, $10 \mathrm{mM}$ sodium succinate, $1 \mathrm{mM}$ DTT, $5 \mathrm{mM}$ ATP, $20 \mathrm{mM}$ HEPES$\mathrm{KOH} \mathrm{pH} \mathrm{7.4).} \mathrm{Import} \mathrm{was} \mathrm{started} \mathrm{with} \mathrm{the} \mathrm{addition} \mathrm{of} 10 \mu \mathrm{L}$ translation product and incubation at $37{ }^{\circ} \mathrm{C}$ for the indicated time. Where indicated, membrane potential was dissipated by treatment with $1 \mu \mathrm{M}$ carbonyl cyanide m-chlorophenyl hydrazone (CCCP) immediately prior to commencement of the experiment. Following protein import, samples were treated as indicated with $50 \mu \mathrm{g} / \mathrm{mL}$ proteinase $\mathrm{K}$ (PK) for 5 minutes followed by treatment with the $1 \mathrm{mM}$ phenylmethylsulfonyl fluoride (PMSF). Mitochondria were collected by centrifugation (12,000 $\mathrm{x} g, 5$ minutes, $4^{\circ} \mathrm{C}$ ) and washed in $100 \mu \mathrm{l}$ import buffer prior to analysis by BN-PAGE and/or SDS-PAGE. Gels were transferred to PVDF as above and dried membranes exposed to phosphor storage screens (GE Healthcare Life Sciences) for 24 hours prior to imaging on a Typhoon ${ }^{\text {TM }} 9400$ Imager (Amersham Sciences).

\section{Mass-spectrometry}

Mitochondria were isolated from HEK293T and NDUFV3 ${ }^{\mathrm{KO}}$ passaged continuously for two weeks in SILAC media as described above. Equal amounts of crude mitochondrial extract were mixed to obtain one replicate HEK293T "light", NDUFV ${ }^{\mathrm{KO}}$ "heavy" and 2 replicates of the reverse orientation. Mixed samples were solubilized with $8 \mathrm{M}$ urea in $50 \mathrm{mM}$ ammonium bicarbonate $(A B C)$ prior to reduction with $5 \mathrm{mM}$ tris-2-carboxyethyl-phosphine (TCEP) incubation at $37^{\circ} \mathrm{C}$ for 30 minutes, followed by alkylation with $50 \mathrm{mM}$ iodoacetamide (IAA). After 30 minutes incubation at room temperature, samples were supplemented with $20 \mathrm{mM}$ DTT and diluted to $2 \mathrm{M}$ urea using $50 \mathrm{mM} \mathrm{ABC}$. Proteins were digested with sequencing 
grade porcine trypsin (Promega) overnight at $37^{\circ} \mathrm{C}$. The digest was then acidified to $1 \%$ trifluoroacetic acid (TFA) and peptides concentrated in a benchtop Centrivap ${ }^{\circledR}$ Centrifugal Vacuum Concentrator. Peptides were desalted using SDB-RPS (3M Empore®) stagetips ${ }^{24}$ made in-house as described ${ }^{15}$. Samples were analyzed using an online high performance liquid chromatography system coupled to a Q- Exactive ${ }^{\text {TM }}$ Plus Hybrid Quadrupole-Orbitrap Mass Spectrometer (Thermo Scientific) as described previously for isolated mitochondria ${ }^{14}$. A single technical re-injection was collected for each sample. Raw files were analyzed using the MaxQuant platform ${ }^{25}$ version 1.5.5.1, searching against the most recent Uniprot human dataset containing reviewed, canonical and isoform variants (August 2016) and a database containing common contaminants by the Andromeda search engine ${ }^{26}$. Default search parameters for an Arg10/Lys8 experiment were used with the following modifications: "Requantify" and "Match between runs" were disabled, and only "Unique" peptides were used for quantification. Intensities for heavy and light peptides were taken from the output "peptides.txt" and a heatmap produced using the Perseus platform ${ }^{27}$ version 1.5.5.3.

\section{Molecular biology}

NDUFV3-S and NDUFV3-L cDNA was obtained following isolation of RNA from control HEK293T cells using TRIzol reagent (Thermofisher). The Superscript III first strand synthesis kit (Thermofisher) was used to generate cDNA primed with oligo(dT), and the resulting product used as template in a PCR primed by oligonucleotides complementary to exons 1 and 4 of NDUFV3. PCR products were ligated into pBABE-puro ${ }^{28}$ (Addgene \#1764) using the NEBuilder HiFi DNA Assembly Master Mix (NEB).

\section{Results and Discussion}

The canonical isoform of NDUFV3 is a $\sim 10 \mathrm{kDa}$ protein that assembles into the distal tip of the complex I matrix arm N-module ${ }^{3,4,14}$. It is one of only three subunits (along with NDUFA7 and NDUFA12) whose loss in gene-edited HEK293T cells resulted in no significant changes in mitochondrial respiration, complex I activity, or the levels of other complex I subunits. Indeed, in NDUFV3 ${ }^{\mathrm{KO}}$ cells, complex I assembles to maturity and only small changes in the mobility of the complex were observed following analysis by blue-native (BN)-PAGE and immunoblotting using antibodies against complex I subunit NDUFA9 (Figure 1A). This shift could be due to loss of the individual protein, however due to the small size of these proteins 
( $\sim 10 \mathrm{kDa})$ relative to the $980 \mathrm{kDa}$ mature complex, we hypothesize the change in migration is due to destabilization of the complex during detergent solubilization and/or electrophoresis and partial dissociation of the $\mathrm{N}$-module ${ }^{14}$.

During our previous proteomic analysis of HEK293T cells ${ }^{14}$, we detected unique peptides that could be attributed to a second isoform of NDUFV3 annotated in the UniProt dataset (P56181-2). Peptides for both this and the canonical isoform of NDUFV3 (P56181) were found at lower levels in those complex I subunit knockout lines where the $\mathrm{N}$-module was also depleted. We confirmed the levels of NDUFV3 peptides in mitochondria isolated from control HEK293T and NDUFV ${ }^{\mathrm{KO}}$ cells using stable isotope labelling with amino acids in cell culture (SILAC) followed by quantitative mass-spectrometry. As seen in Figure 1B, unique peptides derived from both isoforms of NDUFV3 (P56181 and P56181-2) were readily detected in HEK293T but not NDUFV ${ }^{\mathrm{KO}}$ mitochondria. Analysis of the NDUFV3 gene structure (Figure $1 C$ ) revealed the second isoform (hereafter NDUFV3-L) is generated by the gain of an additional exon through alternative splicing, leading to the translation of a $\sim 50 \mathrm{kDa}$ protein. This represents a protein almost five-times larger than the canonical isoform of NDUFV3. To confirm transcription of NDUFV3-L is from the same gene as NDUFV3-S, we generated cDNA from control HEK293T cells and performed PCR using primers complementary to exons 1 and 4 of NDUFV3. As seen in Figure 1D, two products could be detected consistent with the predicted sizes of each splicing variant.

Next, we analyzed the level of NDUFV3 protein in mitochondria from control HEK293T cells by SDS-PAGE and immunoblotting with NDUFV3 antibodies (Figure 2A, lanes 1-2). Two bands migrated at a molecular weight consistent with the predicted sizes of NDUFV3-S and NDUFV3-L and were absent in NDUFV ${ }^{\mathrm{KO}}$ mitochondria. Strikingly, NDUFV3-L appeared to be the dominantly expressed isoform of NDUFV3 in control HEK293T cells. To determine if expression of the NDUFV3 isoforms varied in different tissue types, we also analyzed mitochondria isolated from mouse liver, brain and heart tissue (Figure 2A, lanes 3-5). NDUFV3-L appeared to be the predominant isoform in liver while both forms were at similar levels in heart. The levels of NDUFV3-L were the lowest in brain tissue. Importantly, the protein levels for NDUFV3 isoforms in different mouse tissues were consistent with the relative gene expression of human NDUFV3 isoforms catalogued by the Genotype-Tissue 
Expression (GTEx) project (http://www.gtexportal.org). Taken together, these data suggest a variable tissue specific distribution of NDUFV3 isoforms.

With the mitochondrial genome accounting for just 13 proteins, the vast majority of mitochondrial proteins are instead encoded by nuclear genes, synthesized by cytosolic ribosomes and imported into the organelle ${ }^{7,29}$. To investigate the biogenesis of NDUFV3-L, an in vitro import assay using radiolabeled precursor proteins was performed. When radiolabeled NDUFV3-S and NDUFV3-L precursors were incubated with isolated HEK293T mitochondria, intermediate and mature forms accumulated in a membrane potential dependent $(\Delta \psi)$ manner (Figure $2 \mathrm{~B}$ ). This is consistent with presence of a cleavable mitochondrial pre-sequence, as has been reported for NDUFV3- $S^{23}$. Both processed forms of NDUFV3-S and NDUFV3-L were protected from degradation by proteinase $\mathrm{K}$, which is unable to cross the outer membrane of the mitochondria (compare lanes 1 and 4 ). To assess the assembly of NDUFV3-L into complex I, the import reactions were analyzed by BN-PAGE. As can be seen (Figure 2C), both isoforms assembled into complex I in a time dependent fashion. Next, we sought to determine if NDUFV3-L is present in complex I in vivo. We chose to analyze mitochondria from mouse heart tissue due to clear expression levels of both NDUFV3-S and NDUFV3-L isoforms (Figure 2B, lane 4). We employed two dimensional (2D)PAGE analysis which separates mitochondrial complexes in the first dimension and individual subunits in the second (Figure 2D, top). Immunoblot analysis revealed that both NDUFV3-S and NDUFV3-L were present in complex I (Figure 2B, middle). Supporting this, both isoforms migrated with complex I subunit NDUFS5 (Figure 2B, bottom). Taken together, NDUFV3-L is efficiently imported into mitochondria and assembled into complex I suggesting it is a bona fide complex I subunit.

The recent cryoEM structures of bovine and ovine complex I from heart mitochondria revealed that NDUFV3-S is present at the tip of the matrix arm ${ }^{3,4,13}$. NDUFV3 is not fully resolved in either structure and only modeled for the $\mathrm{N}$-terminal half of the short isoform. As the structures are based on single particle averaging we hypothesize that this lack of resolution may be due to the presence of two populations of complex I harboring one or the other isoform in a similar location. Could NDUFV3-L be accommodated in the structure? While the isoform specific sequence corresponding to exon 3 is predicted to be intrinsically 
unstructured $^{30,31}$, its insertion would be at a solvent exposed location that could be accommodated within the complex I structure without impacting on other subunits (Figure $3 A$, arrow).

Next, we asked if both isoforms are required for complex I assembly. We used CRISPR/Cas9 mediated genome-editing to generate a HEK293T cell line specifically lacking NDUFV3-L through targeting of the isoform-specific third exon (NDUFV3- ${ }^{\mathrm{KO}}$ ). Gene-editing was confirmed by sequencing, revealing two alleles with insertions leading to frame-shift mutations and a subsequent stop codon 24 nucleotides downstream in the case of allele 1 (Figure 3B). Analysis of mitochondria from NDUFV3-L ${ }^{\mathrm{KO}}$ cells confirmed that NDUFV3-L was absent (Figure 3C). In contrast, NDUFV3-S appeared to be present in levels higher than observed for control HEK293T, or a clone harboring unknown mutations (Figure 3C, clone 6). Finally, the state of complex I assembly in mitochondria isolated from NDUFV $3^{\mathrm{KO}}$ and NDUFV3-L ${ }^{\mathrm{KO}}$ was compared by BN-PAGE and immunoblotting (Figure 3D). Unlike in NDUFV $3^{\mathrm{KO}}$ mitochondria, complex I in NDUFV3- $\mathrm{L}^{\mathrm{KO}}$ mitochondria migrated at a similar position to that observed for control mitochondria. Taken together, our data suggests that NDUFV3-S is alone sufficient for the full assembly of complex I.

Finally, we consulted the Exome Aggregation Consortium (ExAC) database ${ }^{32}$ containing the sequenced exomes from $\sim 60,000$ individuals to survey the distribution of NDUFV3 loss-offunction (LoF) mutations in humans. ExAc listed 273 individuals homozygous for one of four different NDUFV3-L specific LoF mutations (Table 1; Figure 1C). In contrast, only heterozygous LoF mutations were detected in the shared second and fourth exons encoding NDUFV3-S. While the large number of individuals harboring homozygous LoF NDUFV3-L mutations suggests that this isoform is not required for normal function of complex I, it should be noted that the splicing of NDUFV3 precludes specific loss of NDUFV3-S. We thus hypothesize that the presence of either isoform may be sufficient at the level of the healthy organism. At present, it is unclear whether individuals lacking NDUFV3-L presented with mitochondrial disease symptoms. However, as the database excluded paediatric patients, who typically represent the largest cohort of affected individuals, it is unlikely that specific loss of NDUFV3-L would be a conventional pathogenic mitochondrial disease mutation. We also cannot exclude the possibility that mutations affecting NDUFV3-L are compensated for 
by increased expression of NDUFV3-S, as we observed in our NDUFV3-L ${ }^{\mathrm{KO}}$ cell line (Figure 3B).

Until now complex IV (cytochrome $c$ oxidase) was the only component of the mammalian respiratory chain known to contain specific isoforms with tissue specific distribution ${ }^{33}$. While differential expression of complex IV subunit isoforms is thought to modulate the enzyme's activity in different cellular environments ${ }^{34}$, the functional role of either isoform of NDUFV3 remains unclear. Its loss in HEK293T cells does not affect the enzymes activity or cellular respiration ${ }^{14}$, making future studies using mouse models essential to elucidation of the proteins function. Inclusion of NDUFV3-L specific knockouts would also permit investigation into the need for dominant expression of that isoform in tissues such as liver.

In conclusion, we report NDUFV3 to be the first complex I subunit expressed in two isoforms with tissue specific distribution. We demonstrate NDUFV3-L to be transcribed, translated and imported into mitochondria where it assembles into complex I with comparable efficiency to that of the canonical NDUFV3 isoform. While both isoforms appear competent to support complex I assembly, the differing stoichiometry of NDUFV3 isoforms in mouse tissues suggests each isoform may have specific roles in complex I function yet to be discovered.

\section{Acknowledgements}

We thank E. Surgenor, L. Twigg, and R. Schittenhelm (Monash Biomedical Proteomics Platform) for assistance. We acknowledge funding from NHMRC Project Grants (1068056, 1107094) and fellowship (1070916 to D.A.S.) and the Australian Mitochondrial Disease Foundation.

\section{References}

1 Lapuente-Brun, E. et al. Supercomplex assembly determines electron flux in the mitochondrial electron transport chain. Science 340, 1567-1570 (2013).

2 Sazanov, L. A. A giant molecular proton pump: structure and mechanism of respiratory complex I. Nat. Rev. Mol. Cell Biol. 16, 375-388 (2015). 
3 Fiedorczuk, K. et al. Atomic structure of the entire mammalian mitochondrial complex I. Nature 538, 406-410 (2016).

4 Zhu, J., Vinothkumar, K. R. \& Hirst, J. Structure of mammalian respiratory complex I. Nature 536, 354-358 (2016).

5 Mimaki, M., Wang, X., McKenzie, M., Thorburn, D. R. \& Ryan, M. T. Understanding mitochondrial complex I assembly in health and disease. Biochim. Biophys. Acta 1817, 851-862 (2012).

6 Andrews, B., Carroll, J., Ding, S., Fearnley, I. M. \& Walker, J. E. Assembly factors for the membrane arm of human complex I. Proc Natl Acad Sci U S A 110, 1893418939 (2013).

7 Alston, C. L., Rocha, M. C., Lax, N. Z., Turnbull, D. M. \& Taylor, R. W. The genetics and pathology of mitochondrial disease. J Pathol (2016).

8 Sanchez-Caballero, L., Guerrero-Castillo, S. \& Nijtmans, L. Unraveling the complexity of mitochondrial complex I assembly: A dynamic process. Biochim Biophys Acta 1857, 980-990 (2016).

9 Zickermann, V. et al. Structural biology. Mechanistic insight from the crystal structure of mitochondrial complex I. Science 347, 44-49 (2015).

10 Vafai, S. B. \& Mootha, V. K. Mitochondrial disorders as windows into an ancient organelle. Nature 491, 374-383 (2012).

11 Miwa, S. et al. Low abundance of the matrix arm of complex I in mitochondria predicts longevity in mice. Nat. Commun. 5, 3837 (2014).

12 Moran, M. et al. Mitochondrial respiratory chain dysfunction: implications in neurodegeneration. Free Radic Biol Med 53, 595-609 (2012).

13 Vinothkumar, K. R., Zhu, J. \& Hirst, J. Architecture of mammalian respiratory complex I. Nature 515, 80-84 (2014).

14 Stroud, D. A. et al. Accessory subunits are integral for assembly and function of human mitochondrial complex I. Nature 538, 123-126 (2016).

15 Stroud, D. A. et al. COA6 is a mitochondrial complex IV assembly factor critical for biogenesis of mtDNA-encoded COX2. Hum Mol Genet 24, 5404-5415 (2015).

16 Formosa, L. E. et al. Characterization of mitochondrial FOXRED1 in the assembly of respiratory chain complex I. Hum. Mol. Genet. 24, 2952-2965 (2015).

17 Montague, T. G., Cruz, J. M., Gagnon, J. A., Church, G. M. \& Valen, E. CHOPCHOP: a CRISPR/Cas9 and TALEN web tool for genome editing. Nucleic Acids Res. 42, W401-407 (2014). 
18 Ran, F. A. et al. Genome engineering using the CRISPR-Cas9 system. Nat. Protoc. 8, 2281-2308 (2013).

19 Reljic, B. \& Stroud, D. A. Screening Strategies for TALEN-Mediated Gene Disruption. Methods Mol Biol 1419, 231-252 (2016).

Acin-Perez, R., Fernandez-Silva, P., Peleato, M. L., Perez-Martos, A. \& Enriquez, J. A. Respiratory active mitochondrial supercomplexes. Mol. Cell 32, 529-539 (2008).

21 Schagger, H. \& von Jagow, G. Tricine-sodium dodecyl sulfate-polyacrylamide gel electrophoresis for the separation of proteins in the range from 1 to $100 \mathrm{kDa}$. Anal. Biochem. 166, 368-379 (1987).

22 McKenzie, M., Lazarou, M., Thorburn, D. R. \& Ryan, M. T. Analysis of mitochondrial subunit assembly into respiratory chain complexes using Blue Native polyacrylamide gel electrophoresis. Anal. Biochem. 364, 128-137 (2007).

23 Lazarou, M., McKenzie, M., Ohtake, A., Thorburn, D. R. \& Ryan, M. T. Analysis of the assembly profiles for mitochondrial- and nuclear-DNA-encoded subunits into complex I. Mol. Cell Biol. 27, 4228-4237 (2007).

24 Rappsilber, J., Mann, M. \& Ishihama, Y. Protocol for micro-purification, enrichment, pre-fractionation and storage of peptides for proteomics using StageTips. Nat Protoc 2, 1896-1906 (2007).

25 Cox, J. \& Mann, M. MaxQuant enables high peptide identification rates, individualized p.p.b.-range mass accuracies and proteome-wide protein quantification. Nat. Biotechnol. 26, 1367-1372 (2008).

26 Cox, J. et al. Andromeda: a peptide search engine integrated into the MaxQuant environment. J. Proteome Res. 10, 1794-1805 (2011).

27 Tyanova, S. et al. The Perseus computational platform for comprehensive analysis of (prote)omics data. Nat Methods 13, 731-740 (2016).

28 Morgenstern, J. P. \& Land, H. Advanced mammalian gene transfer: high titre retroviral vectors with multiple drug selection markers and a complementary helperfree packaging cell line. Nucleic Acids Res. 18, 3587-3596 (1990).

29 Chacinska, A., Koehler, C. M., Milenkovic, D., Lithgow, T. \& Pfanner, N. Importing mitochondrial proteins: machineries and mechanisms. Cell 138, 628-644 (2009).

30 Ishida, T. \& Kinoshita, K. PrDOS: prediction of disordered protein regions from amino acid sequence. Nucleic Acids Res 35, W460-464 (2007). 
31 Dosztanyi, Z., Csizmok, V., Tompa, P. \& Simon, I. IUPred: web server for the prediction of intrinsically unstructured regions of proteins based on estimated energy content. Bioinformatics 21, 3433-3434 (2005).

32 Lek, M. et al. Analysis of protein-coding genetic variation in 60,706 humans. Nature 536, 285-291 (2016).

33 Huttemann, M. et al. Regulation of oxidative phosphorylation, the mitochondrial membrane potential, and their role in human disease. J Bioenerg Biomembr 40, 445456 (2008).

34 Pierron, D. et al. Cytochrome c oxidase: evolution of control via nuclear subunit addition. Biochim Biophys Acta 1817, 590-597 (2012). 


\section{Figure Legends}

Figure 1. A: Mitochondria were isolated from HEK293T and NDUFV3 ${ }^{\mathrm{KO}}$ cell lines, solubilized in 1\% triton X-100 and complexes analyzed by BN-PAGE and immunoblotting with NDUFA9 antibodies detecting complex I (Cl; top panel). The blot was reprobed with SDHA antibodies detecting complex II (CII) as a loading control. (bottom panel). *, non-specific band. B: Mitochondria were isolated from SILAC labeled HEK293T and NDUFV3 ${ }^{\mathrm{KO}}$ cell lines and analyzed by mass-spectrometry as described in the Materials and Methods. Peptides representing NDUFV3-S (P56181) and NDUFV3-L (P56181-2) are shown along with the summed eXtracted ion current (expressed as $\log _{10}$ intensity) of the isotopic cluster belonging to the relevant label pair and amino acid start position of the peptide in the relevant protein. C: Schematic of NDUFV3 gene structure for NDUFV3-S and NDUFV3-L. Loss of function mutations detailed in Table 1 are indicated. D: cDNA was generated from total HEK293T RNA and primers complementary to exons 1 and 4 used to prime PCR amplification of NDUFV3 isoforms.

Figure 2. A: Mitochondria were isolated from HEK293T and NDUFV3 ${ }^{\mathrm{KO}}$ cell lines and C57BL/6 mouse brain, heart and liver tissues. Mitochondrial proteins were separated by SDS-PAGE followed by immunoblotting using antibodies against NDUFV3. *, non-specific bands; OE, over-exposed region of the blot containing NDUFV3-S. B: NDUFV3-S and NDUFV3-L precursor proteins radiolabeled with $\left[{ }^{35} \mathrm{~S}\right]$-methionine were imported into isolated HEK293T mitochondria for the indicated time periods. Prior to import, membrane potential $(\Delta \psi)$ was dissipated by addition of CCCP. Samples were then split and treated with Proteinase K (ProtK) as indicated. Mitochondrial proteins were separated by SDS-PAGE and visualized by digital autoradiography. C: As per B but following import mitochondria were solubilized in 1\% triton X-100 and complexes separated by BN-PAGE. p, precursor; i, intermediate; m, mature; $\mathrm{Cl}$, complex I; Cl/CIII $/ \mathrm{CIV}$, supercomplex. D: Mitochondria were isolated from mouse heart, solubilized in 1\% triton X-100 and complexes separated by BNPAGE. The lane was excised and proteins separated by SDS-PAGE in the second dimension followed by blotting onto PVDF membrane. The blot was stained with Coomassie (top) 
followed by immunoblotting with NDUFV3 (middle panel) or NDUFS5 antibodies (bottom panel). CII, complex II; CIII, complex III; CIV, complex IV; CV, complex V.

Figure 3. A: Location of the $10 \mathrm{kDa}$ subunit (NDUFV3) in the bovine structure of complex I (PDB: 5LDW ${ }^{4}$ ). Inset shows the $10 \mathrm{kDa}$ subunit in cartoon depiction with the $\mathrm{N}$-terminus indicated. Arrow indicates where insertion of exon 3 would occur. IM, inner membrane; IMS, intermembrane space. B: Sequence analysis of NDUFV3 alleles showing deletions in the targeted region from NDUFV3-L ${ }^{\mathrm{KO}}$ cells. C: Mitochondria from control HEK293T, NDUFV ${ }^{\mathrm{KO}}$, NDUFV3-L ${ }^{\mathrm{KO}}$ and a incompletely disrupted clone (clone 6 ) analyzed by SDS-PAGE immunoblotting with NDUFV3 antibodies. D: Mitochondria from control HEK293T, NDUFV ${ }^{\mathrm{KO}}$, and NDUFV3-L ${ }^{\mathrm{KO}}$ were solubilized in $1 \%$ triton $\mathrm{X}-100$ and complexes analyzed by BN-PAGE and immunoblotting using NDUFA9 antibodies. $\mathrm{Cl}$, complex I.

Table 1. Homozygous NDUFV3 loss of function mutations from Exome Aggregation Consortium database ${ }^{a}$.

\begin{tabular}{cccccccc}
\hline Location $^{\text {b }}$ & Exon & Variant & Consequence & Annotation & $\begin{array}{c}\text { Allele } \\
\text { Count }^{\mathbf{c}}\end{array}$ & Homozygotes & $\begin{array}{c}\text { Allele } \\
\text { Frequency }^{\mathbf{d}}\end{array}$ \\
\hline $21: 44323720$ & 3 & $\mathrm{C} \rightarrow \mathrm{T}$ & p.Arg200Ter & Stop Gained & 7535 & 270 & $6.297^{\star} 10^{-2}$ \\
$21: 44323668$ & 3 & $\mathrm{~A} \rightarrow \mathrm{AG}$ & p.Leu185AlafsTer11 & Frameshift & 153 & 1 & $1.275^{\star} 10^{-3}$ \\
$21: 44324336$ & 3 & $\mathrm{AG} \rightarrow \mathrm{A}$ & p.Ala406ProfsTer45 & Frameshift & 2 & 1 & $1.858^{\star} 10^{-5}$ \\
$21: 44324388$ & 3 & $\mathrm{~T} \rightarrow \mathrm{A}$ & C.1264+2T>A & $\begin{array}{c}\text { Splice } \\
\text { Donor }\end{array}$ & 45 & 1 & $9.325^{\star} 10^{-4}$ \\
\hline
\end{tabular}

$\overline{{ }^{a} \text { http://exac.broadinstitute.org }}$

${ }^{b}$ chromosome number:base number

${ }^{c}$ Allele count represents how many times this mutation was found across 60,706 exomes.

dAllele frequency quantifies the frequency of mutant allele from high quality genotype reads. 


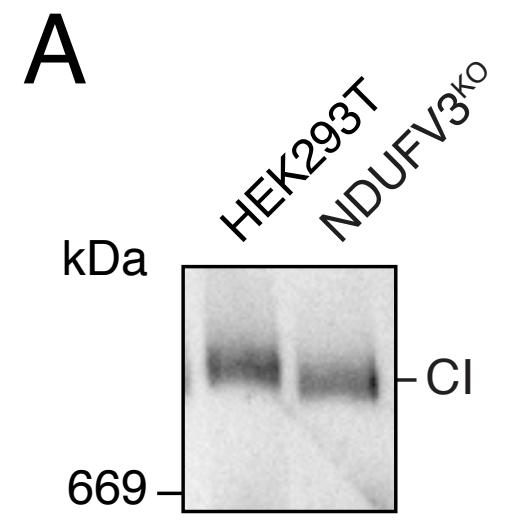

IB:NDUFA9

440

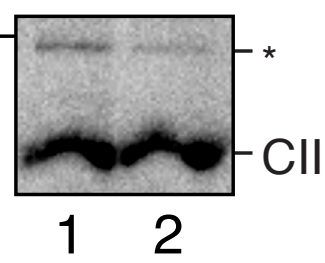

IB:SDHA
B

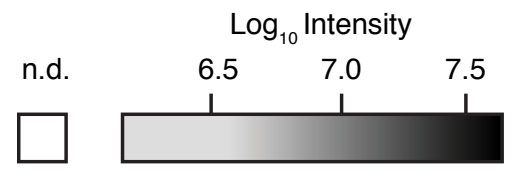

Protein(s)

P56181

P56181-2

P56181-2

P56181-2

P56181-2

P56181-2

P56181-2

P56181-2

P56181-2

P56181-2

P56181-2

P56181-2

P56181-2

P56181-2

P56181-2

$\mathrm{P} 56181 ; \mathrm{P} 56181-2$
(Start position)-Sequence

(57)-KPAPVPAEPFDNTTYK

(67)-LLATQTAAELSK

(79)-NLSSPSSYPPAVNK

(95)-KVASPSPSGSVLFTDEGVPK

(96)-VASPSPSGSVLFTDEGVPK

(187)-KPEASHSFENR

(209)-TLLQKPHVDITDPEKPHQPK

(252)-SQVDEEFLK

(274)-LNEIDKESQKPFEVK

(305)-GSPAPAVLAEEAR

(318)-AEGQLQASPPGAAEGHLEKPVPEPQR

(318)-AEGQLQASPPGAAEGHLEKPVPEPQRK

(352)-KETSGTQGIEGHLK

(400)-LEAEGEAMEDAAAPGDDR

(418)-GGTQEPAPVPAEPFDNTTYK

(438)-NLQHHDYSTYTFLDLNLELSK

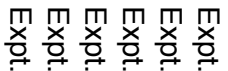

$-N \omega-i \omega$

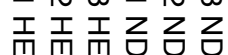

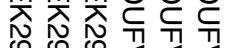

ట్

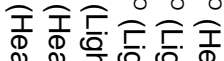
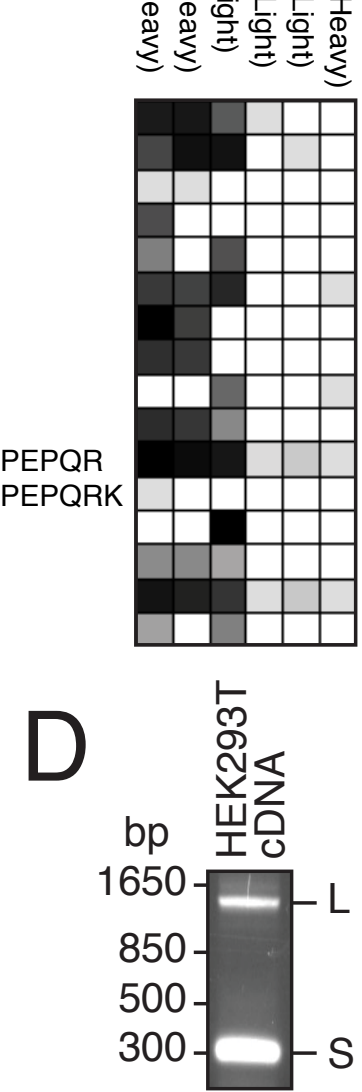

Dibley et al. Figure 1 
A

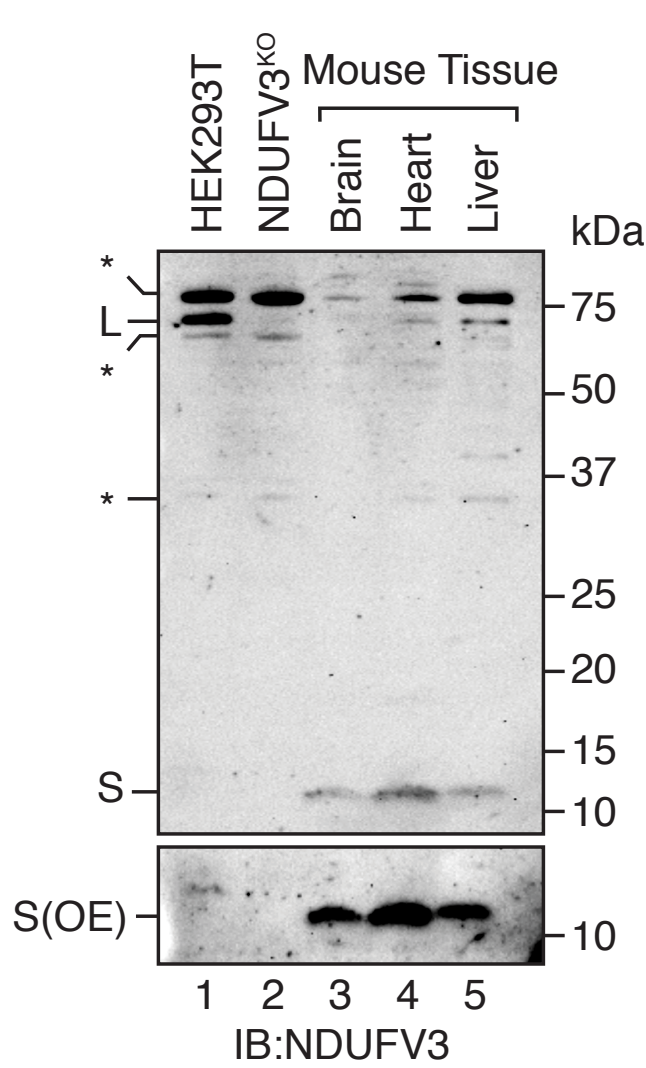

B

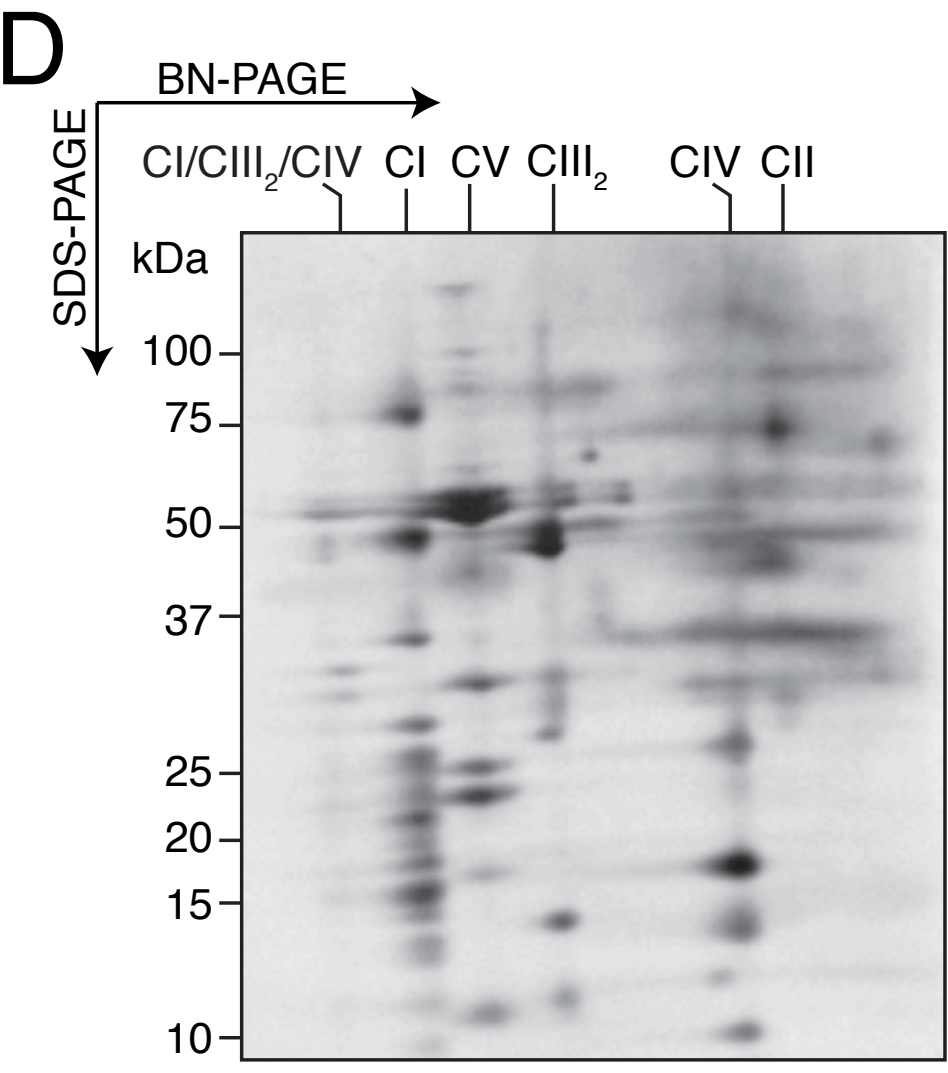

Coomassie

Time (Min.) 5303053030
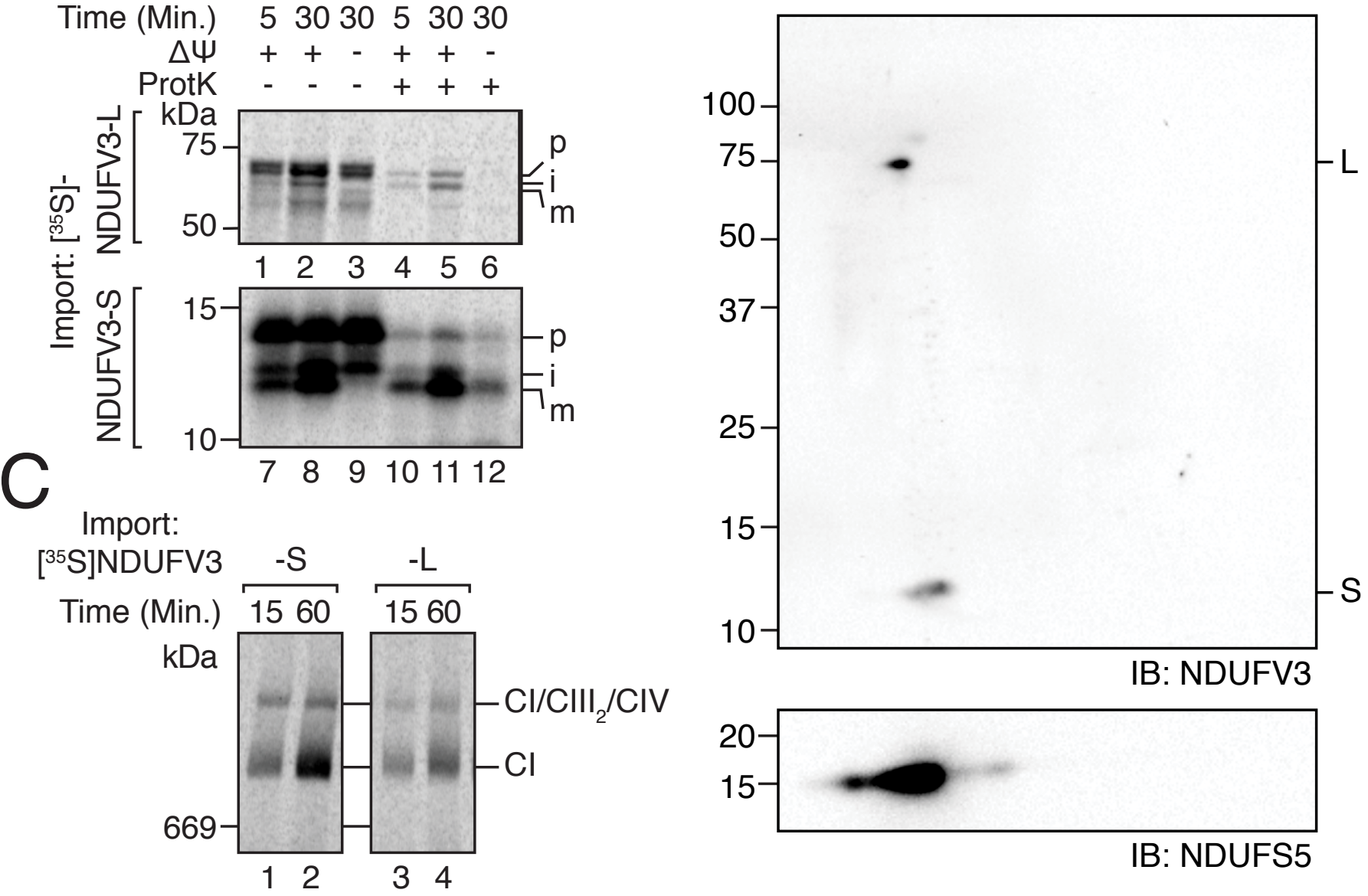

IB: NDUFV3

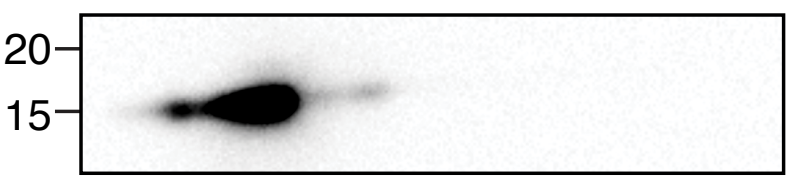

IB: NDUFS5

Dibley et al. Figure 2 


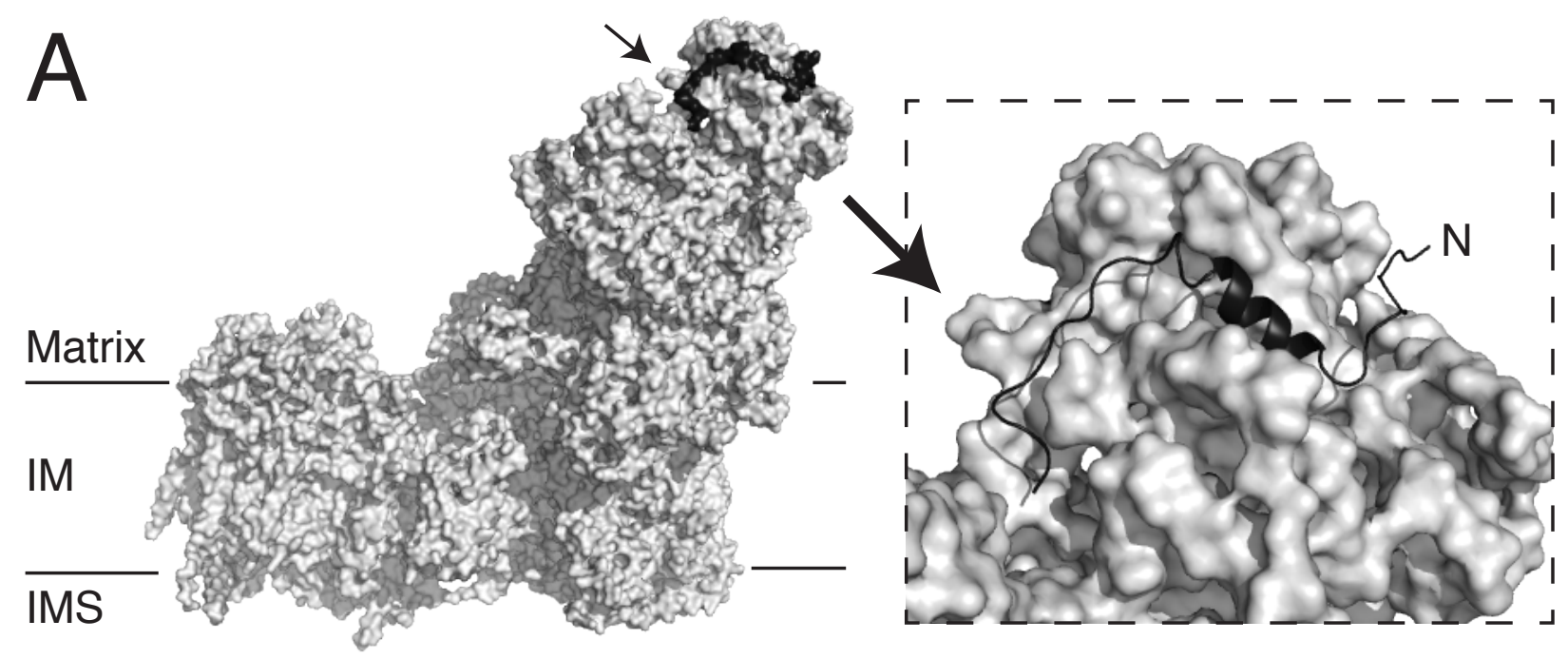

\section{B}

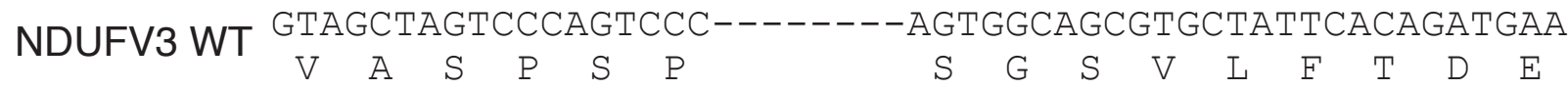
NDUFV3-L-1 GTAGCTAGTCCCAGTCCCC------AGTGGCAGCGTGCTATTCACAGATGAA NDUFV3-L-2 GTAGCTAGTCCCAGTCCCCAGTCCACAGTGGCAGCGTGCTATTCACAGATGAA 21: 42903303 42903333
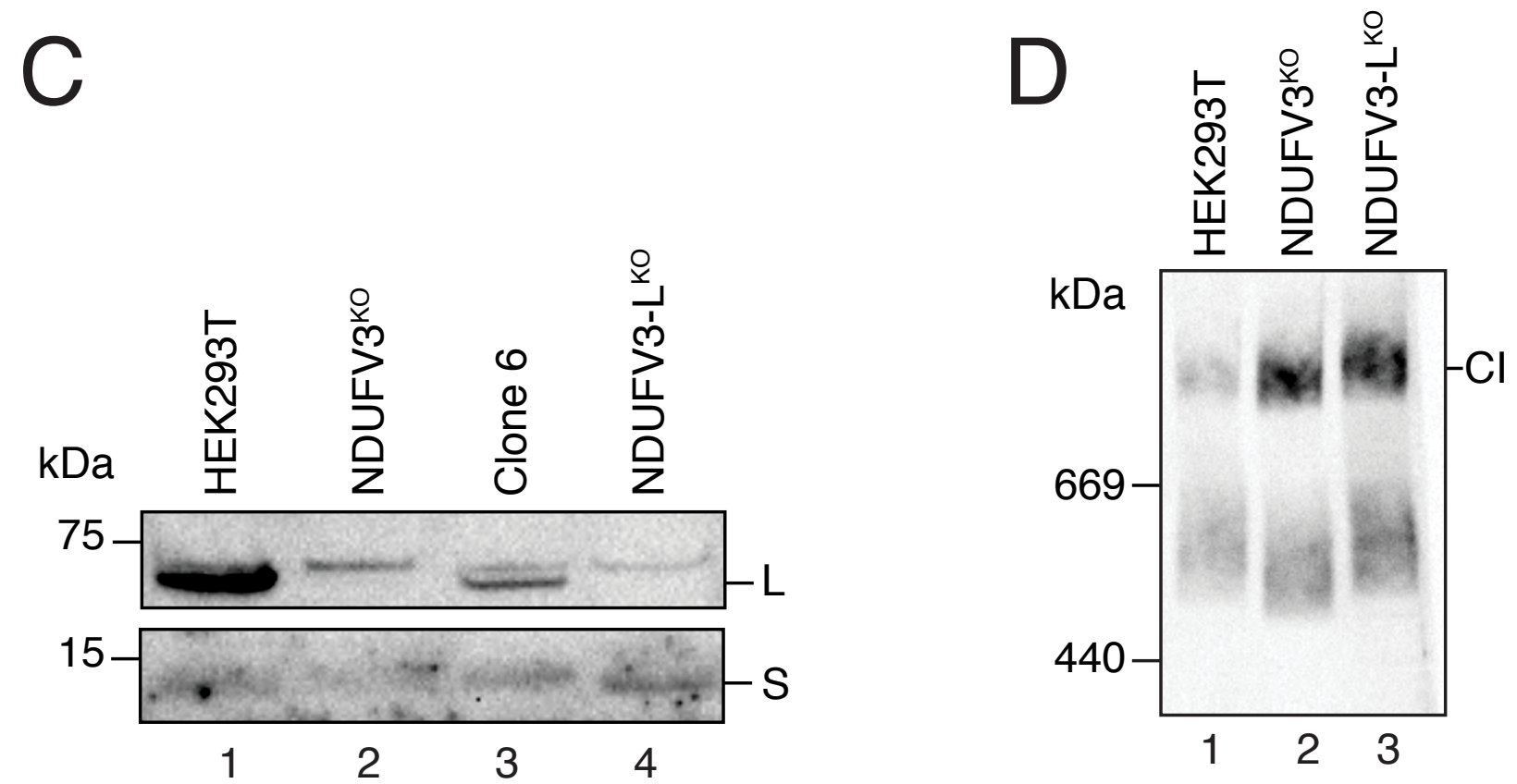

IB: NDUFV3

IB: NDUFA9

Dibley et al. Figure 3 